\title{
Application of EMD-Based SVD and SVM to Coal-Gangue Interface Detection
}

\author{
Wei Liu, Kai He, Qun Gao, and Cheng-yin Liu \\ School of Information and Electronics Engineering, Shandong Institute of Business and Technology, Yantai 264005, China
}

Correspondence should be addressed to Wei Liu; liuweiyyjn@126.com

Received 1 November 2013; Accepted 21 March 2014; Published 14 April 2014

Academic Editor: Feng Gao

Copyright (C) 2014 Wei Liu et al. This is an open access article distributed under the Creative Commons Attribution License, which permits unrestricted use, distribution, and reproduction in any medium, provided the original work is properly cited.

Coal-gangue interface detection during top-coal caving mining is a challenging problem. This paper proposes a new vibration signal analysis approach to detecting the coal-gangue interface based on singular value decomposition (SVD) techniques and support vector machines (SVMs). Due to the nonstationary characteristics in vibration signals of the tail boom support of the longwall mining machine in this complicated environment, the empirical mode decomposition (EMD) is used to decompose the raw vibration signals into a number of intrinsic mode functions (IMFs) by which the initial feature vector matrices can be formed automatically. By applying the SVD algorithm to the initial feature vector matrices, the singular values of matrices can be obtained and used as the input feature vectors of SVMs classifier. The analysis results of vibration signals from the tail boom support of a longwall mining machine show that the method based on EMD, SVD, and SVM is effective for coal-gangue interface detection even when the number of samples is small.

\section{Introduction}

Today a major problem facing the mining industry is how to develop an automated top-coal caving system that can maximize the ratio of coal to gangue. The working procedure of top-coal caving is automatically controlled by an electrohydraulic system, which determines the recovery ratio of top-coal to gangue. In order to improve the recovery ratio of top-coal, a lot of work has been done on coalgangue interface detection (CID) [1-3]. This paper proposes a new CID method based on the analysis of vibration signals due to the collapse of coal and gangue onto the tail boom of a longwall mining machine. Some significant features that differ between coal and gangue can be extracted by analyzing these vibration signals. The acquired vibration signals are usually nonlinear and nonstationary, so it is difficult to effectively extract features. Recently, the timefrequency analysis methods are widely used in the feature extraction of vibration signals $[4,5]$. Among all available time-frequency analysis methods, the wavelet transform may be the best one $[6,7]$. However, wavelet transform is not a selfadaptive signal processing method. Also, energy leakage will occur when wavelet transform is used to process signals, due to the fact that it is an adjustable windowed Fourier transform in nature [8]. The empirical mode decomposition (EMD) decomposes any time-varying signal into its fundamental intrinsic oscillatory modes [9]. The EMD is a self-adaptive time-frequency analysis method that is perfectly applicable to nonlinear and nonstationary processing $[10,11]$.

Recently, singular value decomposition (SVD) of matrix has been widely applied to signal processing, statistical analysis, automatic control, and so forth [12]. According to the matrix theory, singular values have good stability and represent the inherent characteristics of the matrix. That is, when a slight change of matrix elements occurs, the change of matrix singular values is small.

In practice, a large number of samples are usually not available. Support vector machine (SVM) is a new machine learning method developed on the basis of statistical learning theory [13]. SVM can solve the learning problem of a smaller number of samples. Meanwhile, SVM has better generalization than artificial neural network (ANN) and guarantees that the local and global optimal solutions are exactly the same.

In this paper, the SVD technique based on EMD is applied to the feature extraction of vibration signals from 


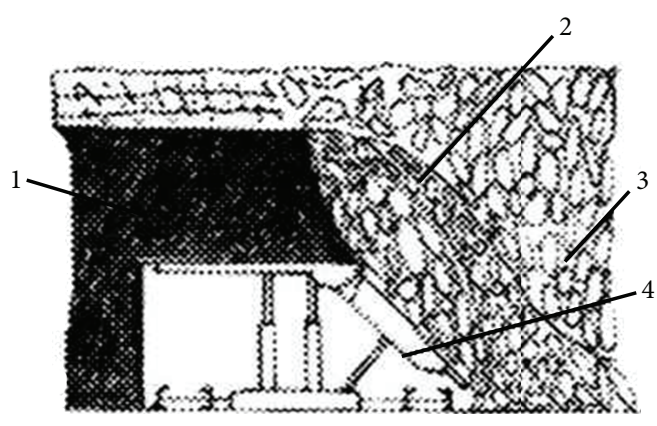

FIGURE 1: Hydraulic support and installation position of sensors (1: coal, 2: coal-gangue, 3: gangue, and 4: sensor).

coal and gangue collapse during top-coal caving. The SVM is introduced into the CID due to its high accuracy and good generalizatio $\mathrm{n}$ for a smaller sample number.

This paper is organized as follows: in the next section, the feature extraction algorithm based on SVD and EMD is discussed. Section 3 briefly reviews the SVM theory. In Section 4, the basic principle of the vibration-based CID experimental system is introduced. The application of the SVM to classification of the caving states is then discussed and the results from a CID validation study are reported. The conclusions are given in the last section.

\section{The SVD Technique Based on EMD}

The EMD is a nonlinear and nonstationary signal analysis method proposed by Huang et al. EMD can decompose any time-varying signal into its fundamental intrinsic mode functions (IMFs), which must satisfy two conditions [9] as follows.

(1) In the whole data set of each intrinsic mode function component, the number of extreme values and the number of zero-crossings must be equal to or differ at most by one.

(2) At any point, the mean value of the envelope defined by local maxima and that defined by the local minima is zero.

With the definition, any time series signal $X(t)$ can be expressed as the sum of the IMF components and the residue

$$
X(t)=\sum_{i=1}^{n} C_{i}(t)+R_{n}(t)
$$

where $C_{i}(t)$ are the IMF components. Here $R_{n}(t)$ is the residue. The IMF includes different frequencies ranging from high to low. Acting as an adaptive data-driven filter bank, the EMD extracts the signal features of disturbances dynamically according to their different physical characteristics.
The IMFs $\left(C_{1}, C_{2}, \ldots, C_{m}\right)$ are chosen to construct the initial feature vector matrix $A$ as follows:

$$
A=\left(\begin{array}{c}
C_{1} \\
C_{2} \\
\vdots \\
C_{m}
\end{array}\right) \in C_{r}^{m \times n}, \quad m \leq n .
$$

Due to the orthogonality of the EMD method, all IMFs are pairwise orthogonal. Therefore, the matrix $A$ must be full rank. By applying the SVD to matrix $A$, then there exists

$$
A=U S V,
$$

where $U \in R^{m \times m}, U U^{\prime}=I ; V \in R^{n \times n}, V V^{\prime}=I ; S=$ $\operatorname{diag}\left\{\lambda_{1}, \lambda_{2}, \ldots, \lambda_{m}\right\}, \lambda_{1} \geq \lambda_{2} \geq \cdots \geq \lambda_{m}$. The values of $\lambda_{i}$ are the singular values of matrix $A$.

As singular values can reflect the nature characteristic of the matrix, the characteristic of vibration signals of coal and gangue can be described effectively by singular values of the initial feature vector matrix. Thus, the singular values of matrix could be used as feature vectors. The SVM could be chosen as the pattern classifier to classify the caving states after the vibration feature vector has been extracted.

\section{Support Vector Machine (SVM)}

As a new generation learning system, SVM enables the nonlinear mapping of an $n$-dimensional input space into a high dimensional feature space. SVM uses a nonlinear mapping to transform an input space to a high-dimension space based on a kernel function and then looks for a nonlinear relation between inputs and outputs in the highdimension space.

Suppose that there is a given training sample set $G=$ $\left\{\left(x_{i}, y_{i}\right), i=1, \ldots, n\right\}$, with each sample $x_{i} \in R^{d}, y_{i} \in\{+1,-1\}$. The classification boundary can be described as follows:

$$
w^{\prime} \cdot x+b=0
$$

where $w$ is a weight vector and $b$ is a bias. Therefore, the following decision function can be used to classify any data set in two classes:

$$
f(x)=\operatorname{sgn}\left(w^{\prime} \cdot x+b\right) .
$$

In order to correctly classify two-class samples, the optimal hyperplane separating the samples can be obtained as a solution to the following constrained optimization problem:

minimize

$$
\varphi(w)=\frac{1}{2}\|w\|^{2}=\frac{1}{2} \mathbf{w}^{T} w
$$

subject to

$$
y_{i}\left[\mathbf{w}^{T} x_{i}+b\right]-1 \geq 0, \quad i=1, \ldots, n .
$$




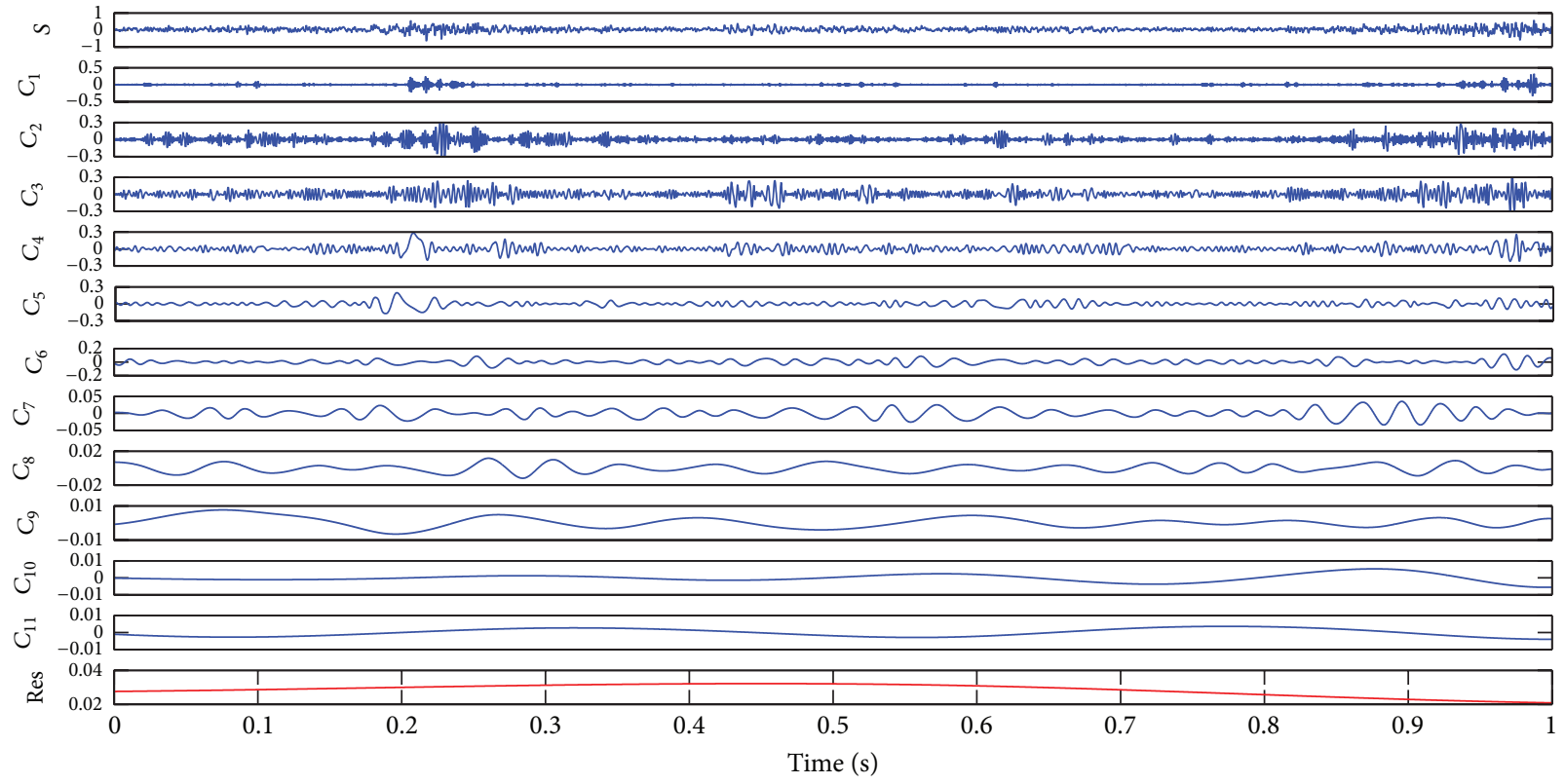

Figure 2: EMD results for top-coal caving.

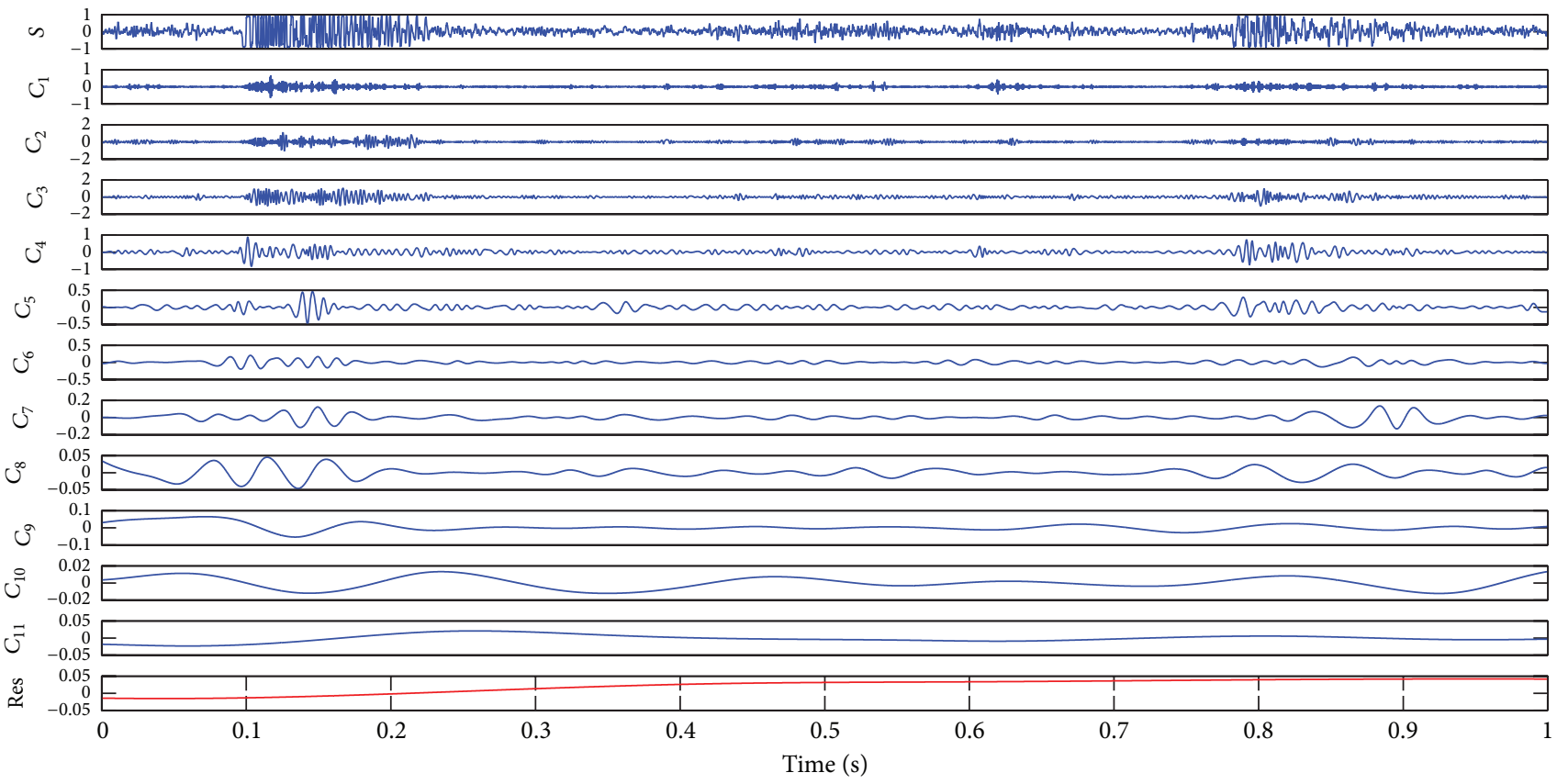

FIGURE 3: EMD results for coal-gangue.

Defining Lagrange multipliers $\alpha_{i} \geq 0$, the optimization problem can be converted to the following:

maximize

$$
P(a)=\sum_{i=1}^{n} a_{i}-\frac{1}{2} \sum_{i=1}^{n} \sum_{j=1}^{n} a_{i} a_{j} y_{i} y_{j}\left(x_{i} \cdot x_{j}\right)
$$

subject to

$$
\alpha_{i} \geq 0, \quad \sum_{i=1}^{n} \alpha_{i} y_{i}=0 .
$$

So the decision function can be expressed as follows:

$$
f(x)=\operatorname{sgn}\left(\sum_{i=1}^{n} a_{i}^{*} y_{i}\left(x_{i} \cdot x\right)+b^{*}\right) .
$$


TABLE 1: Comparison of singular values of selected IMFs for each caving state.

\begin{tabular}{lccccccc}
\hline & $\lambda_{1}$ & $\lambda_{2}$ & $\lambda_{3}$ & $\lambda_{4}$ & $\lambda_{5}$ & $\lambda_{6}$ & $\lambda_{7}$ \\
\hline Top-coal caving & 5.8913 & 5.0226 & 4.7560 & 3.5182 & 2.7658 & 2.6858 & 1.0905 \\
Coal-gangue caving & 19.3695 & 13.7673 & 12.2830 & 6.8331 & 6.5870 & 4.2883 & 3.0371 \\
IMF component & $C_{3}$ & $C_{2}$ & $C_{4}$ & $C_{5}$ & $C_{1}$ & $C_{6}$ & $C_{7}$ \\
\hline
\end{tabular}

TABLE 2: Samples for singular values of IMFs.

\begin{tabular}{|c|c|c|c|c|c|c|c|c|}
\hline Number & $\lambda_{1}$ & $\lambda_{2}$ & $\lambda_{3}$ & $\lambda_{4}$ & $\lambda_{5}$ & $\lambda_{6}$ & $\lambda_{7}$ & Expected output \\
\hline 1 & 6.8036 & 5.1018 & 3.8767 & 2.8366 & 2.1208 & 1.8678 & 1.0817 & $(1,0)$ \\
\hline 2 & 6.1150 & 4.8672 & 4.5894 & 3.3310 & 2.8880 & 2.3330 & 1.2493 & $(1,0)$ \\
\hline 3 & 6.2398 & 5.8762 & 4.1749 & 3.0470 & 2.4142 & 1.9705 & 0.8274 & $(1,0)$ \\
\hline 4 & 5.7488 & 5.2618 & 3.9826 & 3.1724 & 2.6175 & 2.1648 & 1.4194 & $(1,0)$ \\
\hline 5 & 6.9123 & 5.8563 & 4.6258 & 3.2843 & 2.2754 & 2.1075 & 0.9596 & $(1,0)$ \\
\hline 6 & 6.3855 & 5.2633 & 3.6245 & 3.2700 & 2.4509 & 2.1743 & 0.8024 & $(1,0)$ \\
\hline 7 & 8.9377 & 8.6310 & 4.5831 & 4.5361 & 3.1545 & 1.8105 & 0.9570 & $(0,1)$ \\
\hline 8 & 15.2788 & 11.2140 & 7.2233 & 5.1752 & 3.4570 & 1.7460 & 1.0975 & $(\mathbf{0}, \mathbf{1})$ \\
\hline 9 & 19.2038 & 13.6730 & 12.1804 & 6.7688 & 6.5208 & 4.1607 & 2.5960 & $(0,1)$ \\
\hline 10 & 14.5418 & 12.4612 & 8.7011 & 6.1077 & 5.0121 & 3.2906 & 1.6954 & $(\mathbf{0}, \mathbf{1})$ \\
\hline 11 & 22.5342 & 14.4046 & 9.4929 & 5.9841 & 5.3480 & 3.6622 & 2.2097 & $(\mathbf{0}, \mathbf{1})$ \\
\hline 12 & 12.0520 & 10.0045 & 9.6424 & 4.9427 & 4.4479 & 3.5481 & 1.3674 & $(0,1)$ \\
\hline
\end{tabular}

TABLE 3: Classification results of SVM.

\begin{tabular}{lccc}
\hline Caving states & Test samples & Error & Classification accuracy \\
\hline Top-coal caving & 18 & 0 & $100 \%$ \\
Coal-gangue caving & 18 & 0 & $100 \%$ \\
\hline
\end{tabular}

\section{Applications}

In order to investigate the EMD-based SVD technique and SVM as a means of distinguishing between top-coal and gangue caving impacts on the tail boom of a mining machine, an experiment has been carried out on number 2303 working face, Zhangcun Mine, Shanxi, China. The CID experimental system is composed of a data acquisition device, an embedded signal analysis platform, and vibration acceleration sensors, as shown in Figure 1. When coal and gangue fall down and shock the tail boom, the acceleration sensors, which are installed on the hydraulic support, acquire vibration signals from the steel plate [14].

4.1. Feature Extraction of Coal-Gangue Vibration Signals. As an example, two different vibration signals of top-coal caving and coal-gangue caving are chosen for further analysis. The sampling frequency of these signals is $8000 \mathrm{~Hz}$ and the sampling time is one second. Firstly, the EMD is applied to the analysis of separate vibration signals of pure coal and coal-gangue. As shown in Figures 2 and 3, the EMD decomposes the two original signals $S$ into eleven IMFs, which contains important information correlated with the vibration signals of coal and gangue impacts. Obviously, IMFs $C_{1}, C_{2}, C_{3}$, and $C_{4}$ have much higher frequencies than other IMFs. IMFs from $C_{8}$ to $C_{11}$ oscillate so slowly that they only contain very low frequencies, which are composed of clutter or noise, for example, vibration signals caused by mechanical devices themselves. As shown in Figure 3, there are two shock characteristics at the time from $0.1 \mathrm{~s}$ to $0.2 \mathrm{~s}$ and from $0.8 \mathrm{~s}$ to $0.9 \mathrm{~s}$, which correspond with gangue falling events. Meanwhile, the frequency and the amplitude of the first seven IMFs are greater than those of other IMFs during coal-gangue caving. Therefore, the first seven IMFs are selected for further study, and the other IMFs are the resuide accordingly.

For each vibration signal of each caving state, the initial feature vector matrix $A$ can be constructed according to (2). Then SVD is applied to the initial feature vector matrix $A$; namely, $A=U S V$, where $A \in R^{7 \times 8000}, U \in R^{7 \times 7}, S \in$ $R^{7 \times 8000}, V \in R^{8000 \times 8000}$. The singular values $\lambda_{i}$ can be obtained, which are shown in Table 1

$$
\lambda_{i}=\left[\lambda_{1}, \lambda_{2}, \ldots, \lambda_{7}\right]
$$

It can be seen from Table 1 that the singular values of matrix are arranged in descending order of significance. The correspondence between the singular values and IMFs is also given. Especially for the coal-gangue caving, the singular values $\lambda_{1}, \lambda_{2}$, and $\lambda_{3}$ are much bigger than the others, so the singular values could be regarded as the feature vectors to be input to the SVM classifier.

4.2. CID Based on SVM. Actually, the CID is to distinguish two caving states, that is, to solve a two-class pattern classification problem. SVM has the advantage of solving a twoclass problem on the basis of searching for structural risk minimization, even in the case of few learning samples [15]. The new CID method based on SVD, EMD, and SVM is given as follows.

Step 1. Acquire $N$ signals at the sample frequency $f_{s}$ under the condition of top-coal caving and coal-gangue caving, 
TABLE 4: Classification results of SVM under few samples.

\begin{tabular}{|c|c|c|c|c|}
\hline \multirow{2}{*}{$\begin{array}{l}\text { Testing sample number } \\
\text { (from Table 2) }\end{array}$} & \multirow{2}{*}{ Real caving state } & \multicolumn{2}{|c|}{ Distance to optimal hyperplane $H$} & \multirow{2}{*}{ Results } \\
\hline & & 126 training samples & 8 training samples & \\
\hline 5 & Top-coal caving & 0.9473 & 0.6561 & +1 (right) \\
\hline 6 & Top-coal caving & 1.0137 & 0.9807 & +1 (right) \\
\hline 11 & Coal-gangue caving & -1.0223 & -0.4178 & -1 (right) \\
\hline 12 & Coal-gangue caving & -1.0004 & -0.4182 & -1 (right) \\
\hline
\end{tabular}

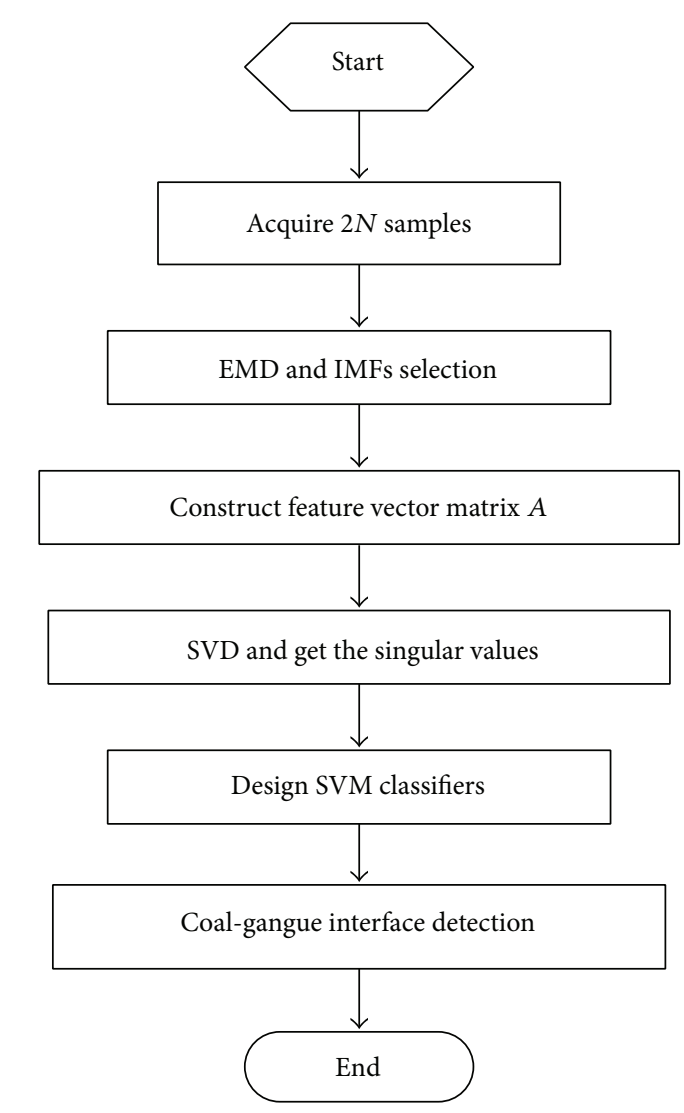

FIgURE 4: The flowchart of EMD-based SVD and SVM.

respectively. The $2 \mathrm{~N}$ samples are divided into two subsets, the training samples sets and the testing samples set.

Step 2. Each signal is decomposed by EMD. Choose the first seven IMFs and construct feature vector matrix $A$. The singular values can be got by applying SVD to matrix $A$.

Step 3. Design SVM classifiers. When the feature input vector is a sample with known state of top-coal caving, the output of SVM classifier is set to 1 , otherwise to -1 . The singular values of the training samples are used as the input to train the SVM classifier. Then the state of caving can be distinguished after the testing samples have been input into the trained SVM classifier.

The flowchart of the proposed method is presented in Figure 4.
4.3. Validation Study. The caving state detection method based on SVD, EMD, and SVM is applied to a vibration sample set of both pure coal and coal-gangue caving. At first, a total of 126 vibration signals are acquired with a sample frequency of $8000 \mathrm{~Hz}, 63$ signals for each caving state. In addition, the testing data sets consisting of 18 signals for each caving are used for validation of this detection method. Then the singular values of each signal are obtained after applying SVD based on EMD, parts of which are listed in Table 2.

Choose RBF kernel function and set $1 / \sigma^{2}=0.5, C=10$. It takes about $0.003 \mathrm{~s}$ to establish the SVM classification model. The number of support vectors is twelve, which accounts for $9.52 \%$ of the total of the training samples. The classification results are shown in Table 3. Obviously, the results are totally consistent with the real caving state.

In order for further study of the classification performance of SVM in the case of a small sample, the number of training samples decreases to eight (number 1 to number 4 and number 7 to number 10 from Table 2 and the rest as testing samples). The classification results are shown in Table 4 . Table 4 shows that the SVM classifier can classify the two caving states accurately even in the case of decreasing the training samples. By comparing the distances between testing samples and the optimal hyperplane $H$, it is found that the overall performance of the SVM classifier weakens as the samples reduce.

\section{Conclusions}

The problem of coal-gangue interface detection (CID) on a fully mechanized mining face has been addressed by applying the SVD technique and EMD to extracting longwall mining machine tail boom support vibration features that can be used for top-coal and coal-gangue caving state classification. EMD is a self-adaptive analysis method that can decompose the signal into a number of IMFs. These functions provide a compact natural representation of nonstationary, nonlinear signals such as those detected by the vibration monitoring of the tail boom support of a longwall mining machine. Singular values were obtained by the application of SVD to the first seven IMFs of the example raw vibration signals (those IMFs containing key feature information), which could be used as the feature input vectors of the classifier. Based on these results, the SVM applied to the singular value vector is proposed as the classification tool for top-coal or coal-gangue caving state. The validation test had a $100 \%$ classification accuracy rate, providing strong support for the robustness of this method. Therefore, the analysis based on SVD, EMD, and 
SVM for longwall mining machine tail boom vibrations offers a new method for CID.

\section{Conflict of Interests}

The authors declare that there is no conflict of interests regarding the publication of this paper.

\section{Acknowledgments}

The work for this paper was supported by the Shandong Provincial Natural Science Foundation of China (Grant nos. ZR2013EEM009 and ZR2013FL019). The authors are grateful to the anonymous reviewers for their careful reviews and detailed comments.

\section{References}

[1] Z.-C. Wang and X.-J. Zhang, "The research on detection of rock content in coal rock mixture in top coal caving by natural gamma ray," Chinese Journal of Sensors and Actuators, vol. 16, no. 4, pp. 442-446, 2003.

[2] G. L. Mowrey, "Horizon control holds key to automation," Coal, vol. 97, pp. 44-49, 1992.

[3] G. L. Mowrey, "New approach to coal interface detection: the in-seam seismic technique," IEEE Transactions on Industry Applications, vol. 24, no. 4, pp. 660-665, 1988.

[4] J. Zou and J. Chen, "A comparative study on time-frequency feature of cracked rotor by Wigner-Ville distribution and wavelet transform," Journal of Sound and Vibration, vol. 276, no. 1-2, pp. 1-11, 2004.

[5] N. Baydar and A. Ball, "A comparative study of acoustic and vibration signals in detection of gear failures using Wigner-Ville distribution," Mechanical Systems and Signal Processing, vol. 15, no. 6, pp. 1091-1107, 2001.

[6] Z. K. Peng and F. L. Chu, "Application of the wavelet transform in machine condition monitoring and fault diagnostics: a review with bibliography," Mechanical Systems and Signal Processing, vol. 18, no. 2, pp. 199-221, 2004.

[7] G. Meltzer and N. P. Dien, "Fault diagnosis in gears operating under non-stationary rotational speed using polar wavelet amplitude maps," Mechanical Systems and Signal Processing, vol. 18, no. 5, pp. 985-992, 2004.

[8] H. T. Vincent, S. L. J. Hu, and Z. Hou, "Damage detection using empirical mode decomposition method and a comparison with wavelet analysis," in Proceedings of the 2nd International Workshop on Structural Health Monitoring, pp. 891-900, Stanford, Calif, USA, 1999.

[9] N. E. Huang, Z. Shen, S. R. Long et al., "The empirical mode decomposition and the Hubert spectrum for nonlinear and non-stationary time series analysis," Proceedings of the Royal Society A: Mathematical, Physical and Engineering Sciences, vol. 454, no. 1971, pp. 903-995, 1998.

[10] N. E. Huang, "Computer implicated empirical mode decomposition method, apparatus, and article of manufacture," Patent US $6311130 \mathrm{B1}, 1996$.

[11] W. Huang, Z. Shen, N. E. Huang, and Y. C. Fung, "Engineering analysis of biological variables: an example of blood pressure over 1 day," Proceedings of the National Academy of Sciences of the United States of America, vol. 95, no. 9, pp. 4816-4821, 1998.
[12] A.-J. van der Veen, E. F. Deprettere, and A. L. Swindlehurst, "Subspace-based signal analysis using singular value decomposition," Proceedings of the IEEE, vol. 81, no. 9, pp. 1277-1308, 1993.

[13] V. Vapnik, The Nature of Statistical Learning Theory, Springer, 2000.

[14] W. Liu, R.-L. Wang, S.-X. Zhang, and Y. Zhang, "Real-time vibration signal acquisition of coal and rock based on DSP and ADS8509," Coal Mine Machinery, vol. 30, pp. 87-199, 2009.

[15] X.-D. Zhang, Modern Signal Processing, Tsinghua University Press, Beijing, China, 2002. 


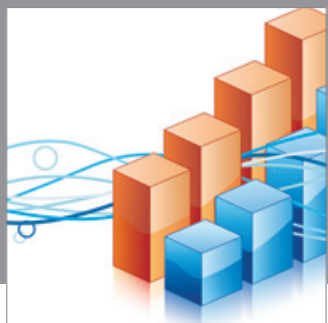

Advances in

Operations Research

mansans

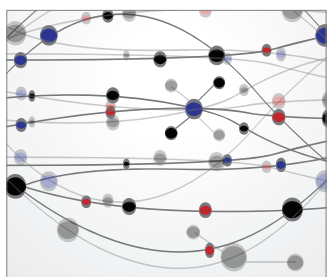

The Scientific World Journal
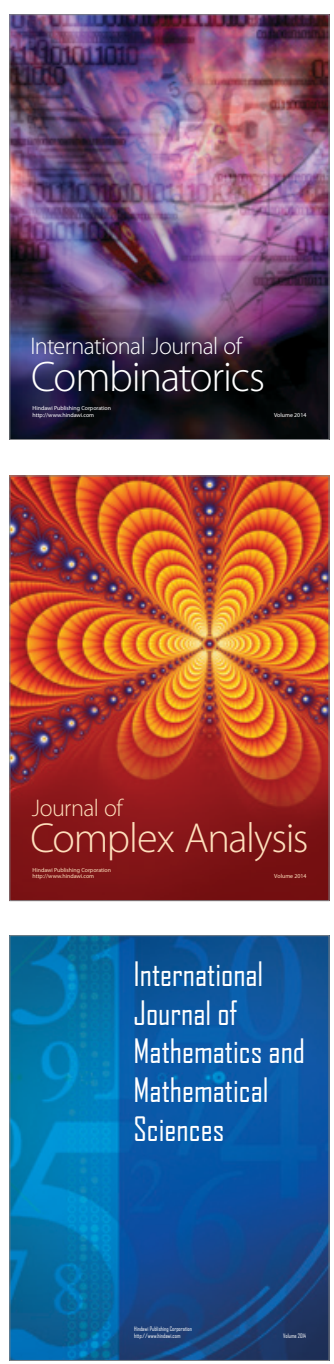
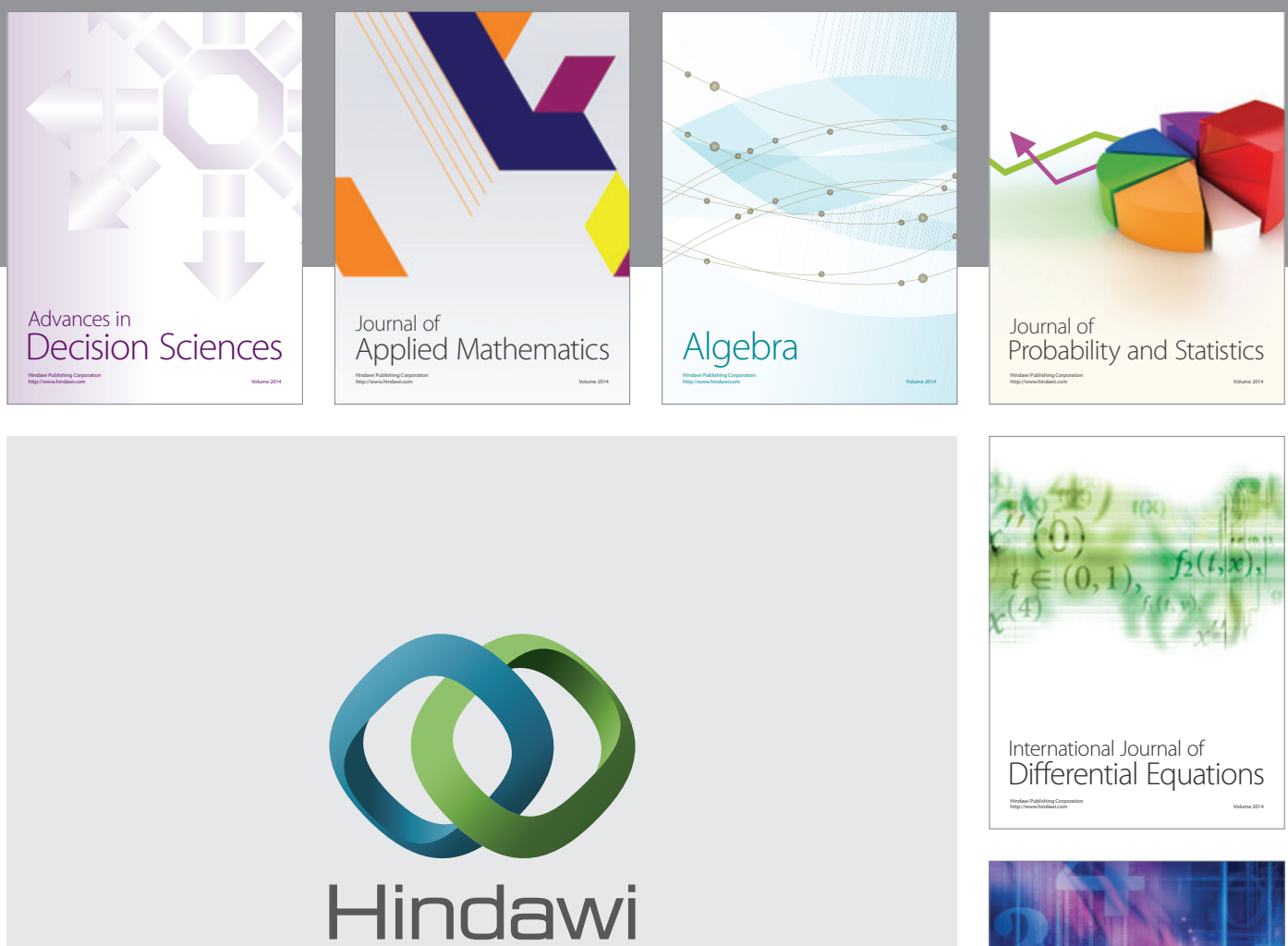

Submit your manuscripts at http://www.hindawi.com
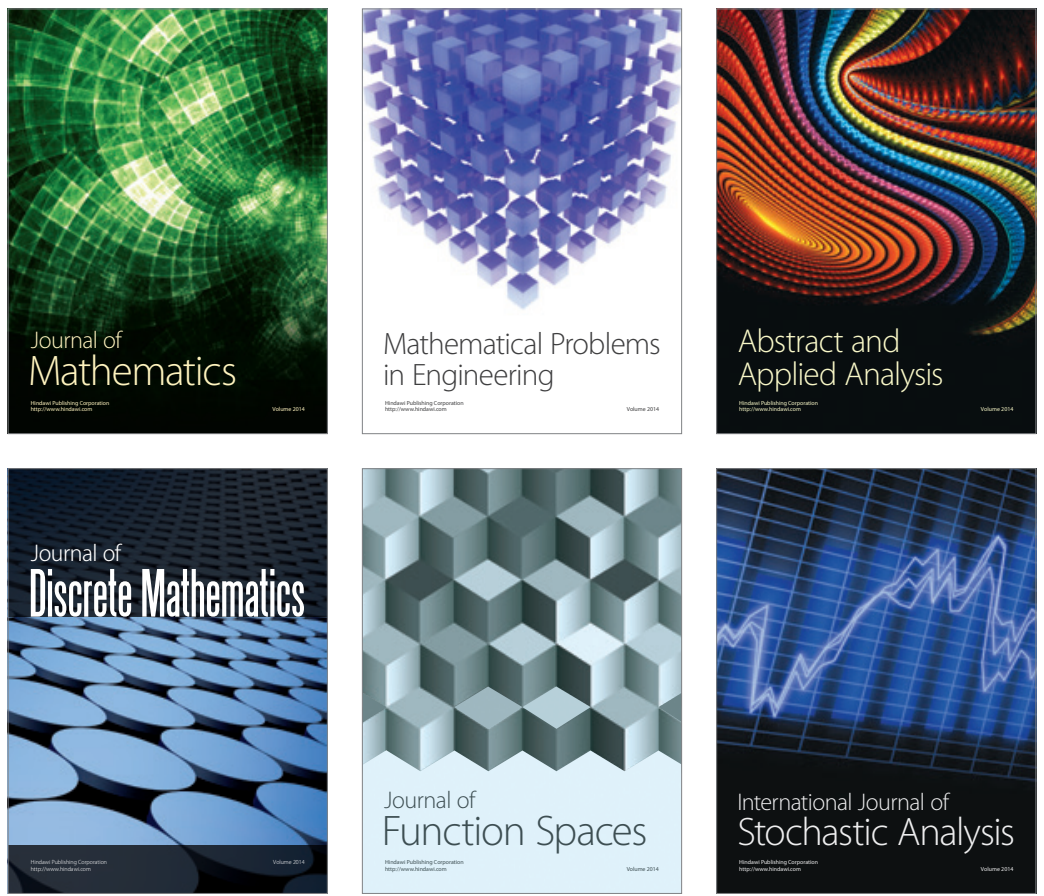

Journal of

Function Spaces

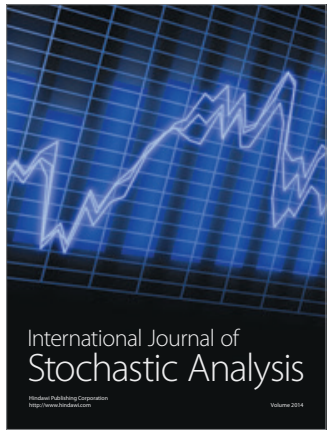

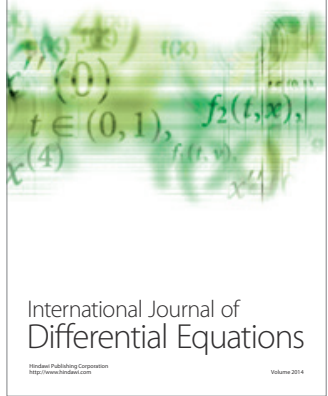
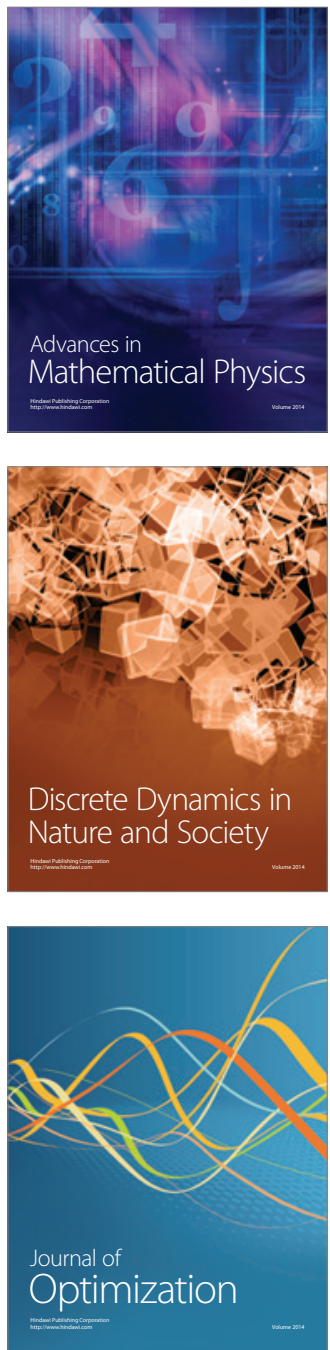